\title{
Preparation and characterization of nanoparticles of carboxymethyl cellulose acetate butyrate containing acyclovir
}

\author{
Venkata Bharadwaz Vedula $\cdot$ Maulick Chopra $\cdot$ \\ Emil Joseph $\cdot$ Sonal Mazumder
}

Received: 23 October 2014/ Accepted: 11 February 2015/Published online: 26 February 2015

(C) The Author(s) 2015. This article is published with open access at Springerlink.com

\begin{abstract}
Nanoparticles of carboxymethyl cellulose acetate butyrate complexed with the poorly soluble antiviral drug acyclovir (ACV) were produced by precipitation process and the formulation process and properties of nanoparticles were investigated. Two different particle synthesis methods were explored-a conventional precipitation method and a rapid precipitation in a multi-inlet vortex mixer. The particles were processed by rotavap followed by freeze-drying. Particle diameters as measured by dynamic light scattering were dependent on the synthesis method used. The conventional precipitation method did not show desired particle size distribution, whereas particles prepared by the mixer showed well-defined particle size $\sim 125-450 \mathrm{~nm}$ before and after freeze-drying, respectively, with narrow polydispersity indices. Fourier transform infrared spectroscopy showed chemical stability and intactness of entrapped drug in the nanoparticles. Differential scanning calorimetry showed that the drug was in amorphous state in the polymer matrix. ACV drug loading was around $10 \mathrm{wt} \%$. The release studies showed increase in solution concentration of drug from the nanoparticles compared to the as-received crystalline drug.
\end{abstract}

Keywords Cellulose · Acyclovir · Nanoparticles · Drug release $\cdot$ Solubility $\cdot$ Reynolds number

\footnotetext{
V. B. Vedula $\cdot$ M. Chopra $\cdot$ S. Mazumder $(\bowtie)$

Department of Chemical Engineering, Birla Institute of Technology and Science (BITS), Pilani 333031, Rajasthan, India e-mail: sonal.mazumder@pilani.bits-pilani.ac.in

E. Joseph

Department of Pharmacy, Birla Institute of Technology and Science (BITS), Pilani 333031, Rajasthan, India
}

\section{Introduction}

Currently there are many options for increasing the bioavailability of poorly soluble drugs like adding of ionized salts, solid dispersions, micronization technique and soft gel technology (Elaine et al. 2003). These methods have their own limitations in terms of drug-loading capacity, toxicity, biodegradability, large dosages and environmental considerations. In the recent years, nanotechnology has emerged as a promising field in addressing these issues (Vivek et al. 2014). One of the most active research areas of nanotechnology is nanomedicine, which applies nanotechnology to highly specific medical interventions for the prevention, diagnosis and treatment of diseases. Currently, nanomedicine is dominated by drug delivery systems, accounting for more than $75 \%$ of total sales (Suwussa et al. 2012).

Herpes virus (HV) which include about 130 different species are potential cause for many viral diseases. Herpes simplex virus-1, the primary virus of all the 25 subfamilies of $\mathrm{HV}$, is the primary cause for genital infections, corneal opacities, shingles and chickenpox in human beings. A recent estimate shows that it infects more than 500 million people worldwide and causes an estimated 23 million new infections every year (Katharine et al. 2008). Transmission of HSV from mother to infant during birth is the most serious complication of genital herpes, and women who acquire HSV during pregnancy are at the highest risk of transmitting the infection (Zane et al. 1997). And also, the risk of HIV acquisition is threefold higher among HSVpositive persons (Esther et al. 2006). Acyclovir (ACV) was approved as drug of choice for treatment of infections caused by this virus (Antona et al. 1994), Fig. 1. ACV is prepared by alkylation of guanine with 2-(chloromethoxy) ethylbenzoate and hydrolyzing the resulting ester. Though 


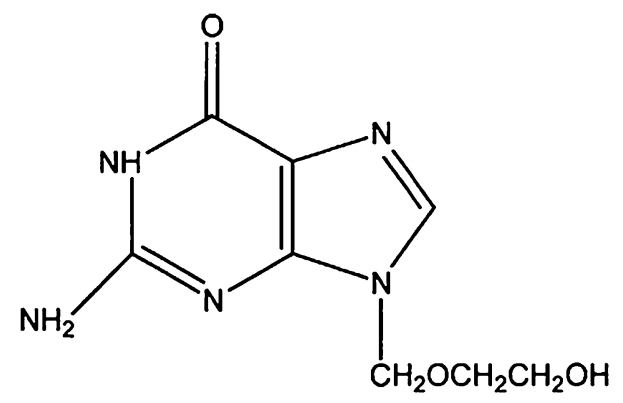

Fig. 1 Structure of acyclovir (David et al. 2013)

ACV is selective and has less cytotoxicity, it has poor bioavailability in the range of $10-30 \%$. This makes its usage difficult if large amounts of dosage are required, necessitating intravenous administration for large concentrations.

To improve its bioavailability, several strategies were developed like transported targeted delivery, where drug is attached to a ligand which is a substrate for specific nutrient transporter, developing analogues of prodrugs, etc., (Ravi et al. 2008). In recent years, much focus has been thrown up on design of new delivery methods for effective administration of antivirals. A number of nanoparticulate systems of ACV like nanoparticles (Bernard et al. 2009; Amany et al. 2009; Florent et al. 2013; Ahmed et al. 2010), nanospheres (Claudia et al. 2003), nanoassemblies (Barbara et al. 2012), metal complexes (Ángel et al. 1999), niosomes (Biswajit et al. 2007; Ismail et al. 2007), nanofibers (Deng-Guang et al. 2011), nanosponges (David et al. 2013) and microparticles (Ga de Jalona et al. 2003) all with the aim of improving bioavailability. However, there is still a lack of understanding in developing a method which can rapidly produce drug-loaded polymeric nanoparticles, at the same time enhancing bioavailability.

Carboxymethyl cellulose acetate butyrate (CMCAB) is a mixed cellulose ester (Fig. 2). It is insoluble in water, water-swellable when partially ionized, but more soluble in common organic solvents than other cellulose esters such as cellulose acetate (CA) or cellulose acetate butyrate
$(\mathrm{CAB})$. It is a stable carrier for metallic pigments. $\mathrm{CMCAB}$ as nanocarriers is one of the thrust areas of research in nanotechnology (Lei et al. 2013). The hydrophobic nature is due to higher degree of substitution (DS) of butyryl and acetyl groups. It is used in oral drug delivery systems as drug particle carrier because of its slow release with respect to time and $\mathrm{pH}$-controlled release of drugs (Junia et al. 2013). Amorphous matrix formulations of CMCAB with different drugs were prepared, and dissolution tests were carried out (Michael et al. 2009; Junia et al. ). These studies showed that CMCAB enhanced the stability of the amorphous drug with respect to crystallization both in solid state and in solution. It has been observed that amorphous drugs in solid dispersions of $\mathrm{CMCAB}$ have greatly enhanced solubility as well as very fast release. Studies conducted to determine the performance of CMCAB in drug delivery with different drug formulations showed zero-order release (Jessica et al. 2007; Li et al. 2013; Bin et al. 2013). The other advantage is that $\mathrm{CMCAB}$-based drug nanoparticles can be prepared very easily through different methods like co-precipitation, spray drying and film casting because of its good solubility in organic solvents.

Considerable work has been done to formulate polymerantiviral drug nanoparticles with synthetic or semisynthetic polymers (Dembri et al. 2001; Löbenberg et al. 1998; Destache et al. 2009; Mainardes et al. 2009; Sharma and Garg 2010; Duan et al. 2010). More recently, studies have shown that polysaccharides are very promising for oral drug delivery due to their affinity for complexing with a variety of drugs which can suppress drug crystallization, their relatively high glass transition temperatures and their biocompatibility (Sandra 2009). The composition and morphology of the polymer matrix play an extremely important role in the drug release and pharmacokinetics (Raval et al. 2011). Nanoparticles have the ability to improve the dissolution rate of the drug (Mainardes and Evangelista 2005). All of these benefits can contribute to the reduction in dose and dosing frequency, thereby reducing the side effects and improving the patient compliance (Hariharan et al. 2004).
Fig. 2 Structure of carboxymethyl cellulose acetate butyrate (CMCAB) (Michael et al. 2009)

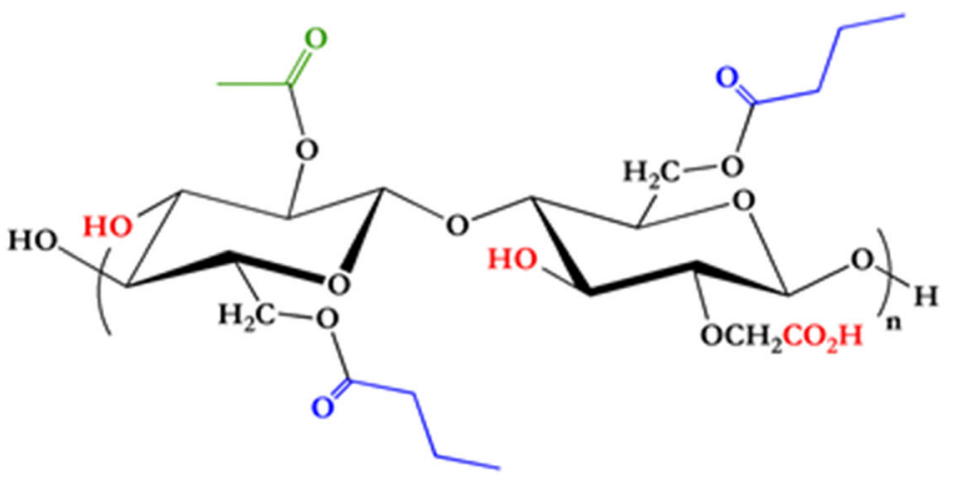

DS Ac $=0.44$

DS $\mathrm{Bu}=1.64$

DS CM $=0.33$ 
The aim of this work is to synthesize and develop ACVCMCAB drug nanoparticles that will have much higher solubility concentration compared to pure drugs when taken orally. There is no previous study reported where these drugs were complexed with CMCAB to form nanoparticles. In this work, polymer drug nanoparticles are prepared through simple precipitation method and flash precipitation method using a multi-inlet vortex mixer (MIVM). The particle sizes and drug loading of the nanoparticles were measured to test control and reproducibility of the particle formation process. The suppression of crystallinity of the drugs in the amorphous nanoparticles was studied by differential scanning calorimetry. Release of the drugs from the polymer matrix was measured to understand the effect of particle size on drug release. Additionally, Reynolds numbers in the mixer were varied to allow rapid and complete mixing leading to controlled particle size.

\section{Experimental procedure}

\section{Materials}

Acyclovir (ACV) was a gift sample from the Department of Pharmacy at BITS Pilani, India. Carboxymethyl cellulose acetate butyrate (CMCAB; approximate molecular weight 22,000 , degree of substitution of butyryl group $=1.64$, degree of substitution of acetyl group $=0.44$, degree of substitution of carboxymethyl group $=0.33$ ) was from Eastman Chemical Company, USA. CMCAB was provided in its free acid form and used as received. Tetrahydrofuran (THF, analytical reagent grade, MOLYCHEM, India) was used for polymer drug nanoparticles formation. Sodium hydroxide (laboratory reagent grade, RFCL, Ankleshwar, India) and potassium di-hydrogen orthophosphate (analytical reagent grade, Qualigen Chemicals, Mumbai) were used for release studies. Mannitol was from $\mathrm{CDH}$, New Delhi, India, and used as a cryoprotectant. Millipore water (18.2 $\mathrm{M} \Omega \bullet \mathrm{cm}$ at $25^{\circ} \mathrm{C}$ ultrapure) was used for all the experiments.

\section{Preparation of ACV-CMCAB nanoparticles}

In this work, polymer drug nanoparticles were prepared by two different methods. First method was a simple precipitation process, and the second method was a rapid precipitation in a multi-inlet vortex mixer.

\section{Simple precipitation}

Two different phases (aqueous and organic) were required for this method. Aqueous phase was prepared by dissolving pure $\mathrm{ACV}$ in distilled water. Mass concentration of
ACV was $0.3703 \mathrm{mg} / \mathrm{ml}$ in distilled water. Organic solution was prepared by adding CMCAB to THF. Polymer concentration in organic phase was $10 \mathrm{mg} / \mathrm{ml}$. Mass ratio of drug to polymer was maintained at 1:3, and volumetric ratio of $\mathrm{THF}$ to water was kept at 1:9. Solutions were stirred at room temperature to dissolve the polymer and drug completely in their respective solvents. Drop-wise method was followed for generation of nanoparticles, i.e., organic phase solution was added drop-wise into the aqueous solution. The final solution consisted of nanoparticles of drug-loaded polymer, free polymer and free drug. THF was removed using a rotary evaporator (BUCHI made, Model: R210a). THF-free solution was then centrifuged for removal of water in a high-speed centrifuge at $13,000 \mathrm{rpm}$ and $20^{\circ} \mathrm{C}$. After centrifugation, particles were kept in an incubator for nearly $15 \mathrm{~h}$ to maintain temperature of $37 \pm 0.5^{\circ} \mathrm{C}$. Retained moisture was removed from the sample by keeping it in oven @ $37 \pm 0.5^{\circ} \mathrm{C}$ for a period of $15-20 \mathrm{~h}$.

\section{Flash precipitation in multi-inlet vortex mixer}

Flash nanoprecipitation of CMCAB drug complexes was carried out in a four-jet multi-inlet vortex mixer that accommodates four streams (Hak-Kim and Philip 2011; Yanxiang et al. 2013; Janine et al. 2009; Boris et al. 2010). The THF solution of CMCAB was injected into the mixer along with three other water streams, where one of the water streams was composed of the drug (ACV). The four inlet streams are tangential to the mixing chamber, and exit stream comes from the center of the chamber. The Reynolds number required for the formation of nanoparticles was investigated. The Reynolds number is a dimensionless number that is a measure of turbulence and, in this case, of mixing time. It depends upon the inlet stream velocities, viscosities and densities (Ying et al. 2008).

The setup consisted of the mixer containing four streams (one organic and three aqueous streams). The streams were connected to two syringe pumps (New Era, Unigenetics, India), one for aqueous (NE-1600) and one for organic (NE-300), respectively. The pumps maintain the flow rate of the streams, and the Reynolds numbers were calculated based on stream flow rates and velocities. In this experimental work, DISPOVAN ${ }^{\mathrm{TM}}$ syringes were used. Schematic of MIVM is shown in Fig. 3.

Mass concentration ratios were kept constant as in simple precipitation method, i.e., $10 \mathrm{mg} / \mathrm{ml}$ of CMCAB in THF and $0.3703 \mathrm{mg} / \mathrm{ml}$ of $\mathrm{ACV}$ in water. Flow rates were controlled by syringe pumps so that the mass concentration of the solution was maintained constant. Reynolds numbers were varied in the range of 2200-17,800 depending on the flow rates. 


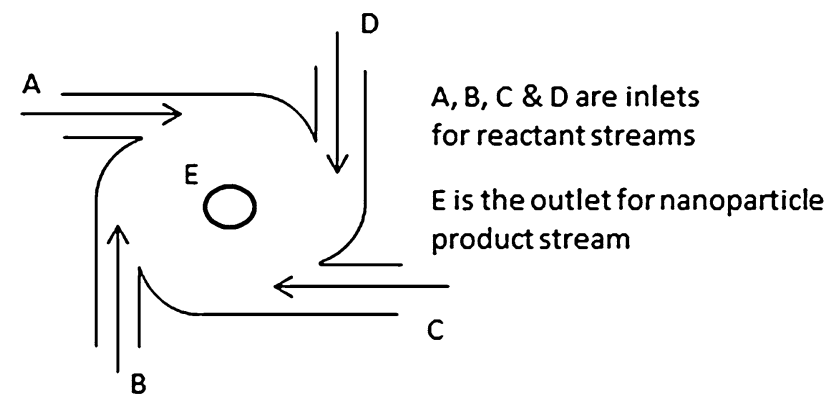

Fig. 3 Schematic representation of MIVM

The nanoparticle solution thus formed was collected and THF was removed using a rotary evaporator at a bath temperature of $60{ }^{\circ} \mathrm{C}$ and 357 mbar pressure. This pressure corresponds to the boiling point of THF at $60{ }^{\circ} \mathrm{C}$. The rotavapped suspension was dried in a LABCONCO freeze dryer at $0.45-0.52$ mbar for 2 days.

\section{Reynolds number experiment}

The Reynolds number was varied six times by varying the aqueous stream flow rates. The organic stream volumetric flow rate was kept constant at $5.82 \mathrm{ml} / \mathrm{min}$. The Reynolds number was calculated by Eq. 1, and the results for six different Reynolds number are shown in Table 1.

$R_{e}=D_{\text {mixer }} \sum \frac{u_{i}}{v_{i}}=D_{\text {mixer }}\left(\frac{u_{1}}{v_{1}}+\frac{u_{2}}{v_{2}}+\frac{u_{3}}{v_{3}}+\frac{u_{4}}{v_{4}}\right)$

where $u_{i}$ is the velocity of each individual inlet stream, and $v_{i}$ is the kinematic viscosity (density over viscosity) of each inlet solvent at the mixer temperature. The volumetric flow rate, $Q_{i}$, is determined by the equation:

$Q_{i}=u_{i} A_{\text {is }}$

where $A_{\text {is }}$ is the cross-sectional area of the inlet streams. When $A_{\text {is }}$ is the same for all four streams and it is assumed that the solution formed by mixing is ideal, i.e., there is no volume change upon mixing.

\section{Nanoparticles drying: effect of cryoprotectant}

It was observed during preparation of first few batches of nanoparticles, that there was significant agglomeration and change in particle size after freeze-drying. During freezedrying, formation of ice crystals causes damage to the basic physiochemical properties of nanoparticles. Upon adding cryoprotectant, the damage is prevented to some extent. Firstly, cryoprotectant partially solubilizes the nanoparticles so that they are less prone to puncture and secondly it interrupts the lattice formation of ice so that the formation of crystals is controlled. In order to control particle size during freeze-drying, a suitable cryoprotectant mannitol was used and the effect on particle size was studied. The suspension after rotavap was divided into two batches. In the first batch, nearly $15 \%(\mathrm{w} / \mathrm{w})$ of mannitol was added as cryoprotectant. The second batch did not have any mannitol. Both the samples were freeze-dried for 2 days to ensure complete moisture removal at temperature of $-50{ }^{\circ} \mathrm{C}$ and $0.45-0.52 \mathrm{mbar}$ vacuum. Particle size was measured after freeze-drying to determine the effect of cryoprotectant on nanoparticles.

Drug loading

In order to measure the drug loading of the nanoparticles, a calibration curve for the ACV dissolved in distilled water was developed using a UV-Visible spectrophotometer (Systronics, 119) at a wavelength of $253 \mathrm{~nm}$, with a bandwidth of $2 \mathrm{~nm}$ and accuracy of $\pm 0.1 \mathrm{~nm}$ within the range of 200-1000 $\mathrm{nm}$ and $10 \mathrm{~mm}$ size cuvettes were used. ACV stock solution was prepared for pure drug standard curve by adding $10 \mathrm{mg}$ of drug in $10 \mathrm{ml}$ distilled water and shaking on auto shaker for 10-15 min. Dilute solutions were prepared from the stock solution at different drug concentrations, viz. 2, 4, 6, 8 and $10 \mu \mathrm{g} / \mathrm{ml}$. The absorbance for resultant dilute solutions was measured at this maximum wavelength and was plotted against concentration to obtain the regression equation relating both concentration and absorbance. An average of three scans was taken for each absorbance value. The standard curve obtained is shown in Fig. 4.

The resulting equation for the calibration curve of rifampicin in acetonitrile was:

Absorbance at $253 \mathrm{~nm}=0.00716 C_{\mathrm{acv}}+0.2632$

where $R^{2}=0.9985$.

To determine the percentage of drug loaded in the nanoparticles, two sample solutions at different concentrations were prepared. Distilled water was used as dilution medium. In the first sample, $1 \mathrm{mg}$ of nanoparticles was dissolved in $10 \mathrm{ml}$ of distilled water, and in the second case, same amount of nanoparticles was dissolved in $20 \mathrm{ml}$. Drug loading of the nanoparticles prepared by both the method was measured using UV-Visible spectrophotometer using the developed calibration curve. Maximum wavelength was set at $253 \mathrm{~nm}$. The absorbance obtained was substituted in the regression equation of standard curve data, and the unknown experimental concentration $\left(C_{\text {drug }}\right)$ was determined. The drug loading $\left(W_{\mathrm{ACV}}\right)$ was calculated from experimental concentration divided by the actual concentration $\left(C_{\text {nanoparticles }}\right)$ as given by Eq. 4 .

$$
\begin{aligned}
W_{\mathrm{ACV}}= & \frac{\text { Final concentration of the ACV in the solvent }}{\text { Initial concentration of particles in the solvent }} \\
& \times 100 \\
= & \frac{C_{\mathrm{ACV}}}{C_{\mathrm{part}}} \times 100
\end{aligned}
$$




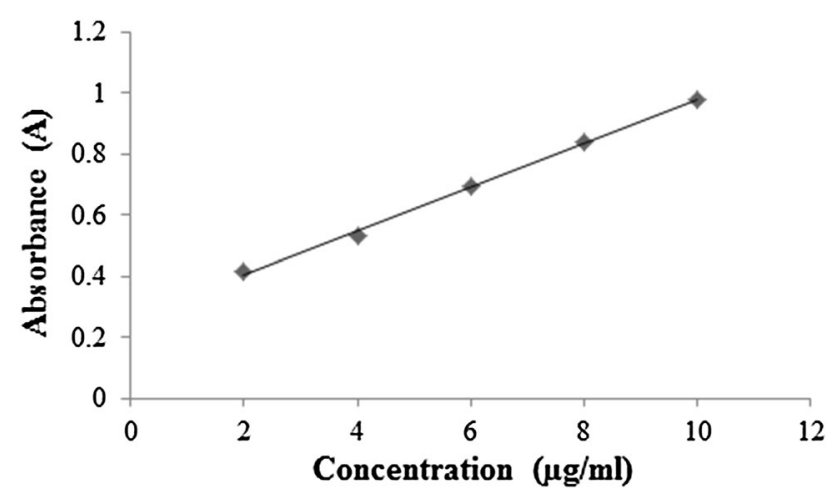

Fig. 4 Calibration curve of acyclovir dissolved in deionized water at $253 \mathrm{~nm}$

$C_{\mathrm{ACV}}=$ Concentration of ACV calculated from calibration curve Eq. 3. $C_{\text {part }}=$ Concentration of the particles dissolved in DI water.

Determination of size, polydispersity index and zeta potential of nanoparticles

Particle size and polydispersity index were found by dynamic light scattering technique using Zetasizer (ModelZEN 3602, Malvern Corp, UK) equipped with Zetasizer software. The measurements were made at a fixed light scattering angle of $173^{\circ}$. Distilled water was used as dispersing solvent for dilution before every measurement. Zeta potential measurements were also conducted in the same cuvette cells. Smoluchowski approximation was selected for $f(\mathrm{Ka})$ parameter as particles are dispersed in aqueous medium. Temperature for all the measurements was set as $25{ }^{\circ} \mathrm{C}$. All measurements were carried out for 12 times for each sample.

Fourier transform infrared (FT-IR) spectroscopy

Fourier transform spectroscopy was carried on ACV, $\mathrm{CMCAB}$ and polymer drug nanoparticles to understand whether any structural differences exist between pure compounds and different nanoparticle systems using Perkin Elmer (Frontier-IR model) spectrophotometer. All particle testings were recorded in the wave number range of $400-4000 \mathrm{~cm}^{-1}$. A total of 10 scans were carried for every measurement.

\section{Differential scanning calorimetry}

To understand the phase transition behavior of the nanoparticles, ACV, CMCAB and ACV-CMCAB nanoparticles were subjected to differential scanning calorimetric analysis (Perkin Elmer, DSC-4000, 2013). The machine was calibrated using indium for heat of fusion and melting point. Range of temperature was set between -70 and $300{ }^{\circ} \mathrm{C}$ at the rate of $10{ }^{\circ} \mathrm{C} / \mathrm{min}$. Standard aluminum pans were used for sampling. An empty sample pan was used as reference. Nitrogen gas flow rate was maintained $20 \mathrm{ml} / \mathrm{min}$ throughout the measurements.

In vitro studies

Typically, equivalent amount of free drug and nanoparticles was dispersed in $80 \mathrm{~mL}$ of potassium phosphate buffer, $\mathrm{pH} 6.8$ (corresponds to the $\mathrm{pH}$ of human small intestine) for $8 \mathrm{~h}$ at $37^{\circ} \mathrm{C}$ (This study which was previously done for $5 \mathrm{~h}$ is repeated for extended time $-8 \mathrm{~h}$. However, the residence time of a particle in the small intestine is approximately $4 \mathrm{~h}$. The previous study was conducted for $5 \mathrm{~h}$ to account for the maximum release of the drug during the residence time of the particle. However, the reviewer's suggestion was noted and the study was repeated for $8 \mathrm{~h}$. It was observed that maximum drug release occurred by fifth hour and no significant release was observed after that). Buffer solution was prepared by adding $0.2 \mathrm{M} \mathrm{NaOH}$ dropwise to $0.2 \mathrm{M}$ potassium phosphate monobasic until the solution $\mathrm{pH}$ was 6.8 . Eighty milliliters of this buffer solution was taken in a beaker and the nanoparticles were dissolved and kept under stirring at $37{ }^{\circ} \mathrm{C}$ to determine the release of nanoparticles. Five milliliters of samples was taken at time intervals of 5, $30 \mathrm{~min}, 1 \mathrm{~h}, 1.5,2,3,45,6,7$ and $8 \mathrm{~h}$. After each time of taking the sample, equal amount of fresh buffer has been added to maintain the sink conditions. Samples were then centrifuged to remove unbound polymer particles from the sample solution. Released ACV from nanoparticles was determined spectrophotometrically at $253 \mathrm{~nm}$. The concentration was then obtained from previously drawn standard plot of ACV in the range of $2-10 \mu \mathrm{g} / \mathrm{ml}$. Released amount was calculated as

$\%$ Release $=\frac{\text { Released Acyclovir }}{\text { Total Acyclovir }}$

\section{Results and discussions}

We investigated how different particle processing schemes affected particle properties, notably particle size and drug loading. These processing approaches included nanoparticle preparation by (1) simple precipitation followed by rotavap, centrifugation and drying in oven and (2) rapid precipitation in a mixer followed by rotavap and freezedrying. We explored the processing conditions on particle size and size distribution. We investigated the drug content, size and crystallinity of the nanoparticles. Drug release studies were conducted with ACV nanoparticles and were compared to pure crystalline drug. The role of CMCAB in enhancing solubility and inhibiting drug crystallinity in the 
particles is also discussed. The multi-inlet vortex mixer makes it possible to produce nanoparticles with well-controlled particle size distributions compared to typical coprecipitation process. Addition of cryoprotectant also improved the particle size distribution of the nanoparticles after processing. This is the first study for preparing nanoparticles of $\mathrm{ACV}$ and $\mathrm{CMCAB}$ by rapid precipitation.

\section{Effect of Reynolds number on particle size}

The main purpose of the experiment was to optimize the Reynolds number for synthesizing well-defined nanoparticles. It was observed that lower Reynolds number gave small particle size and narrow polydispersity index (Fig. 5). There was an increase in the polydispersity index of the particles (Table 1). The reason could be that at higher Reynolds numbers, nucleation of particles increased and there was no enough time for the interaction of drug and the polymer. Hence aggregates with uncontrolled particle sizes were obtained. Additionally, zeta potentials of the particles were also observed. The magnitude of the

\section{Particle Size vs. Reynolds Number}

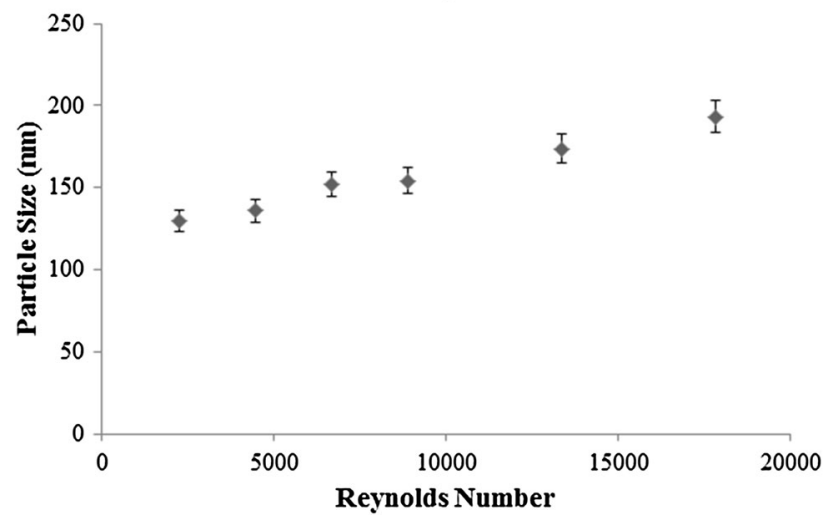

Fig. 5 Particle sizes versus Reynolds number of three batches of ACV-CMCAB nanoparticles after fabrication in the multi-inlet vortex mixer

Table 1 Particle size analysis, polydispersity and zeta potential of ACV-CMCAB particles after preparing them in MIVM at varying Reynolds Number (2224-17792) by DLS-The average results from three batches are shown

\begin{tabular}{lclll}
\hline S. no & $\begin{array}{l}\text { Reynolds } \\
\text { number }\end{array}$ & $\begin{array}{l}\text { Particle } \\
\text { size }(\mathrm{nm})\end{array}$ & $\begin{array}{l}\text { Polydispersity } \\
\text { index }\end{array}$ & $\begin{array}{l}\text { Zeta potential } \\
(\mathrm{mV})(\mathrm{SD} \pm 3)\end{array}$ \\
\hline 1 & 2224 & 129.8 & 0.209 & -41.7 \\
2 & 4444 & 135.8 & 0.209 & -47.3 \\
3 & 6664 & 151.8 & 0.327 & -51.1 \\
4 & 8888 & 154.3 & 0.366 & -47.9 \\
5 & 13,340 & 173.8 & 0.412 & -45.6 \\
6 & 17,792 & 193.2 & 0.434 & -44.9 \\
\hline
\end{tabular}

zeta potential is predictive of the colloidal stability. Nanoparticles with zeta potential values greater than $+25 \mathrm{mV}$ or less than $-25 \mathrm{mV}$ typically have high degrees of stability. Dispersions with a low zeta potential value will eventually aggregate due to Van Der Waal inter-particle attractions. Zeta potential experiments were performed to analyze the state of nanoparticle surface or whether the processing steps have modified the nanoparticle surface. The results showed that the particles were stable in solution irrespective of their sizes. All the experiments were conducted three times for each set of Reynolds number and the percentage error was approximately $5 \%$.

Well-defined particle size, polydispersity index and zeta potential were obtained at Reynolds number $\sim 2200$. So, all the further characterization techniques, cryoprotectant effect and release studies were conducted for particles prepared at Reynolds number 2200.

Particle size, polydispersity index and zeta potential

The particle size measurement revealed lower size and polydispersity indices for particles synthesized by the flash nanoprecipitation method. The size of the particles obtained by simple precipitation method was large $\sim 810 \mathrm{~nm}$ with polydispersity index of 0.71 . The effect of cryoprotectant on the final particle size after freeze-drying was significant as shown in Fig. 6. Significant agglomeration was observed during freeze-drying process as observed in the sample without cryoprotectant. Several theories have been proposed such as crystal bridge theory, capillary pressure theory, hydrogen bond theory and chemical bond theory to explain the agglomeration phenomenon (Wang et al. 2002). The aggregation of particles in this work is possibly due to the ice crystal formation repelling foreign particles away from the interstitials. During sublimation, the ice crystals leave microscopic pores. Another cause can be during drying of water, loss of electrostatic stabilization leads to particle-particle aggregation. Also, nanoparticles become attached as a result of hydrogen bond and/or

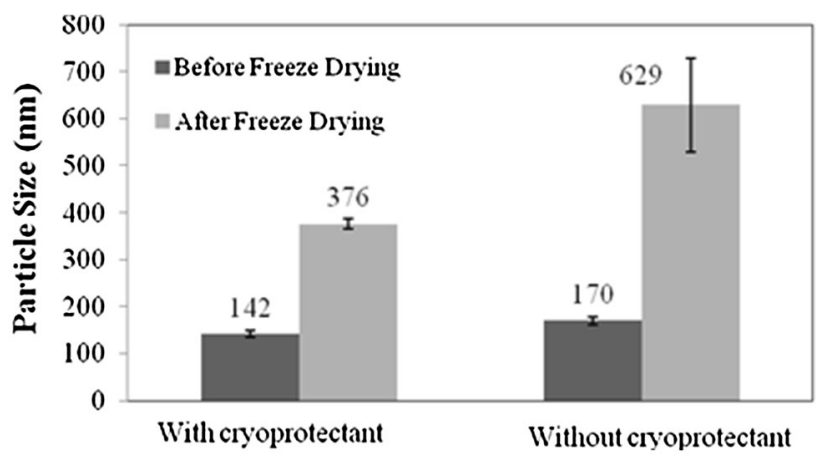

Fig. 6 Effect of cryoprotectant effect on nanoparticle size before and after freeze-drying 
bridging of surface hydroxyl groups. This can be minimized by using cryoprotectors such as glucose, sucrose, trehalose, mannitol. In this study, addition of mannitol inhibited particle agglomeration during freeze-drying (Table 2). Further investigations with larger amount of mannitol will be necessary to obtain well-defined particle size with narrow polydispersity index. The effect of cryoprotectant and the particle size (or polydispersity) did not affect the values of zeta potential. In all cases, the zeta potential analysis revealed colloidal stability of the nanoparticles.

Fourier transform infrared (FT-IR) spectroscopy study

FT-IR spectra of ACV, CMCAB and ACV-CMCAB nanoparticles are shown in Figs. 7, 8 and 9, respectively. The analyses of peaks were based on previous studies (Ben 1997; Barbara 2004). For pure ACV, characteristic peaks were observed because of $\mathrm{O}-\mathrm{H}$ bend at $1103 \mathrm{~cm}^{-1}, \mathrm{C}-\mathrm{O}$ stretch at $1047.9 \mathrm{~cm}^{-1}, \mathrm{CH}_{2}$ wagging at $901.52 \mathrm{~cm}^{-1}$ and $\mathrm{CH}_{2}$ stretch and $\mathrm{NH}$ wagging at $681.99 \mathrm{~cm}^{-1}$. OH stretch because of free moisture at $3440 \mathrm{~cm}^{-1}$ and a peak at $3178 \mathrm{~cm}^{-1}$ corresponding to $\mathrm{CH}$ aromatic ring were observed. For CMCAB, peaks were noticed at $2965 \mathrm{~cm}^{-1}$

Table 2 Effect of cryoprotectant addition (during freeze-drying) on size of nanoparticle prepared by flash nanoprecipitation in MIVM at Re 2200

\begin{tabular}{lrrrrl}
\hline Parameter & \multicolumn{2}{c}{ With cryoprotectant } & & \multicolumn{2}{l}{ Without cryoprotectant } \\
\cline { 2 - 3 } \cline { 5 - 6 } \cline { 5 - 6 } & Before & After & & Before & After \\
\hline Particle size $(\mathrm{nm})$ & 142.1 & 376.3 & & 169.8 & 629 \\
Polydispersity index & 0.2 & 0.4 & & 0.2 & 0.6 \\
Zeta potential $(\mathrm{mV})$ & -52.5 & -62.6 & & -59.4 & -69.1 \\
\hline
\end{tabular}

because of $\mathrm{COOH}$ dimer group, $\mathrm{C}=\mathrm{O}$ stretch of $\mathrm{CHO}$ group at $1732.37 \mathrm{~cm}^{-1}, \mathrm{O}-\mathrm{H}$ bend at $1159.93 \mathrm{~cm}^{-1}$ and $\mathrm{C}-\mathrm{C}$ skeletal vibration at $1037.7 \mathrm{~cm}^{-1}$.

For the case of ACV-CMCAB nanoparticles both with and without mannitol (Fig. 9), common characteristic peaks similar to that of $\mathrm{CMCAB}$ were noticed like $\mathrm{C}=\mathrm{O}$ stretch of CHO group at 1738.49 and $1732.52 \mathrm{~cm}^{-1}$, respectively. Similarly, characteristic peaks of ACV were observed at 679.19 and $679.12 \mathrm{~cm}^{-1}$, respectively, in both the nanoparticles due to $\mathrm{NH}$ wagging. The other peaks found in nanoparticles without mannitol presence are at $1161.03 \mathrm{~cm}^{-1}$ due to $\mathrm{CO}$ stretch and at $1059.71 \mathrm{~cm}^{-1}$ because of $\mathrm{C}-\mathrm{C}$ skeletal vibration. It can be noted that this $\mathrm{C}-\mathrm{C}$ skeletal vibration and $\mathrm{CO}$ stretch in nanoparticles are present though not exactly at the same wave numbers as in that of CMCAB and ACV.

In the case of nanoparticles with mannitol addition, three additional peaks were observed. One because of $\mathrm{C}=\mathrm{C}$ stretch at $1629.56 \mathrm{~cm}^{-1}, \mathrm{C}-\mathrm{H}$ bending at $1366 \mathrm{~cm}^{-1}$ and the other at $1228.36 \mathrm{~cm}^{-1}$ because of COC asymmetric stretch (Peng and Thomas 2008). From the basic chemical structure of mannitol, there was no presence of any $\mathrm{C}=\mathrm{C}$ bonds. So, it has come from $\mathrm{C}=\mathrm{C}$ aromatic ring of $\mathrm{ACV}$. The source for other peak, i.e., COC asymmetric stretch at $1228.36 \mathrm{~cm}^{-1}$ is unclear. It can be either from out of plane COC bonds of CMCAB or from COC bonds from mannitol. Similar is the case for $\mathrm{C}-\mathrm{H}$ bending at $1366 \mathrm{~cm}^{-1}$. It is unclear whether the peak is from $\mathrm{CH}$ bonds of $\mathrm{CMCAB}$ or mannitol or ACV.

In the spectrum of nanoparticles, no peaks were observed in the range from 4000 to $1800 \mathrm{~cm}^{-1}$. The spectrum is nearly stable which indicates that no transmittance of incident infrared waves has taken place in this region.

As the particles were coated with $\mathrm{CMCAB}$, the free hydroxyl bond and $\mathrm{CH}$ aromatic rings of $\mathrm{ACV}$ may be
Fig. 7 FT-IR spectra of asreceived $\mathrm{ACV}$

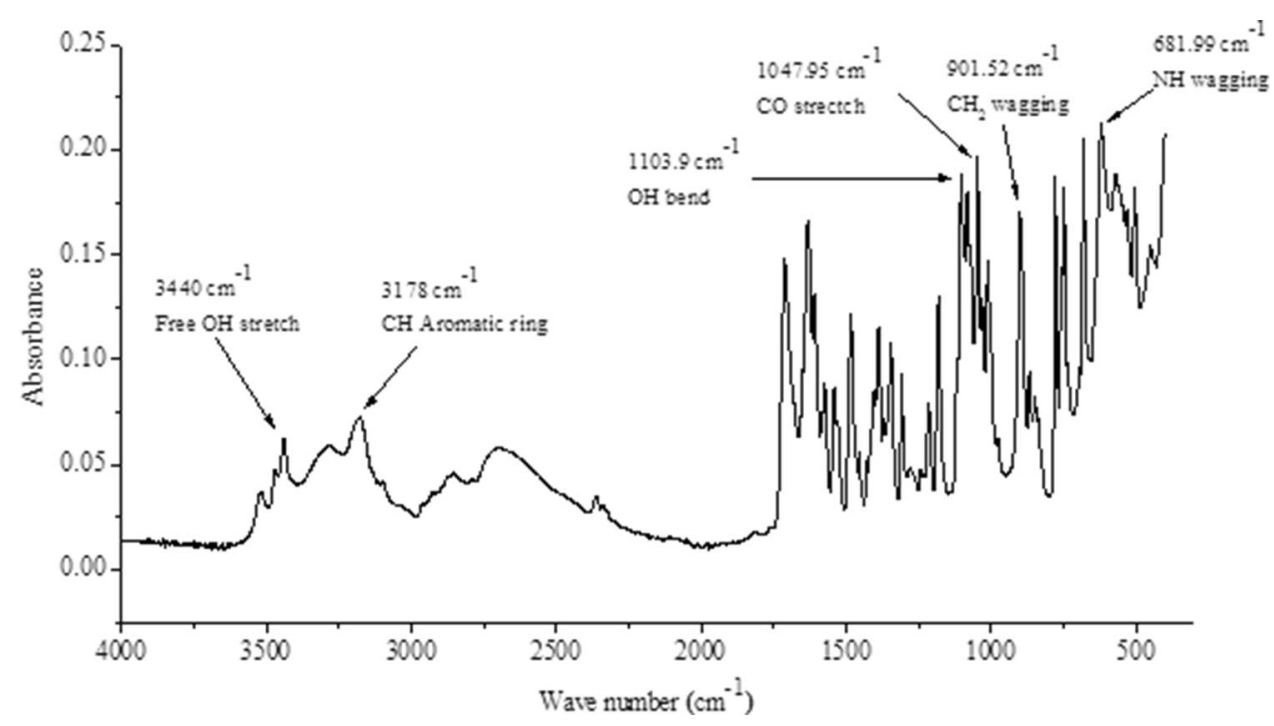

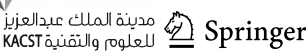


Fig. 8 FT-IR spectra of pure CMCAB

Fig. 9 FT-IR spectra of ACV$\mathrm{CMCAB}$ nanoparticles
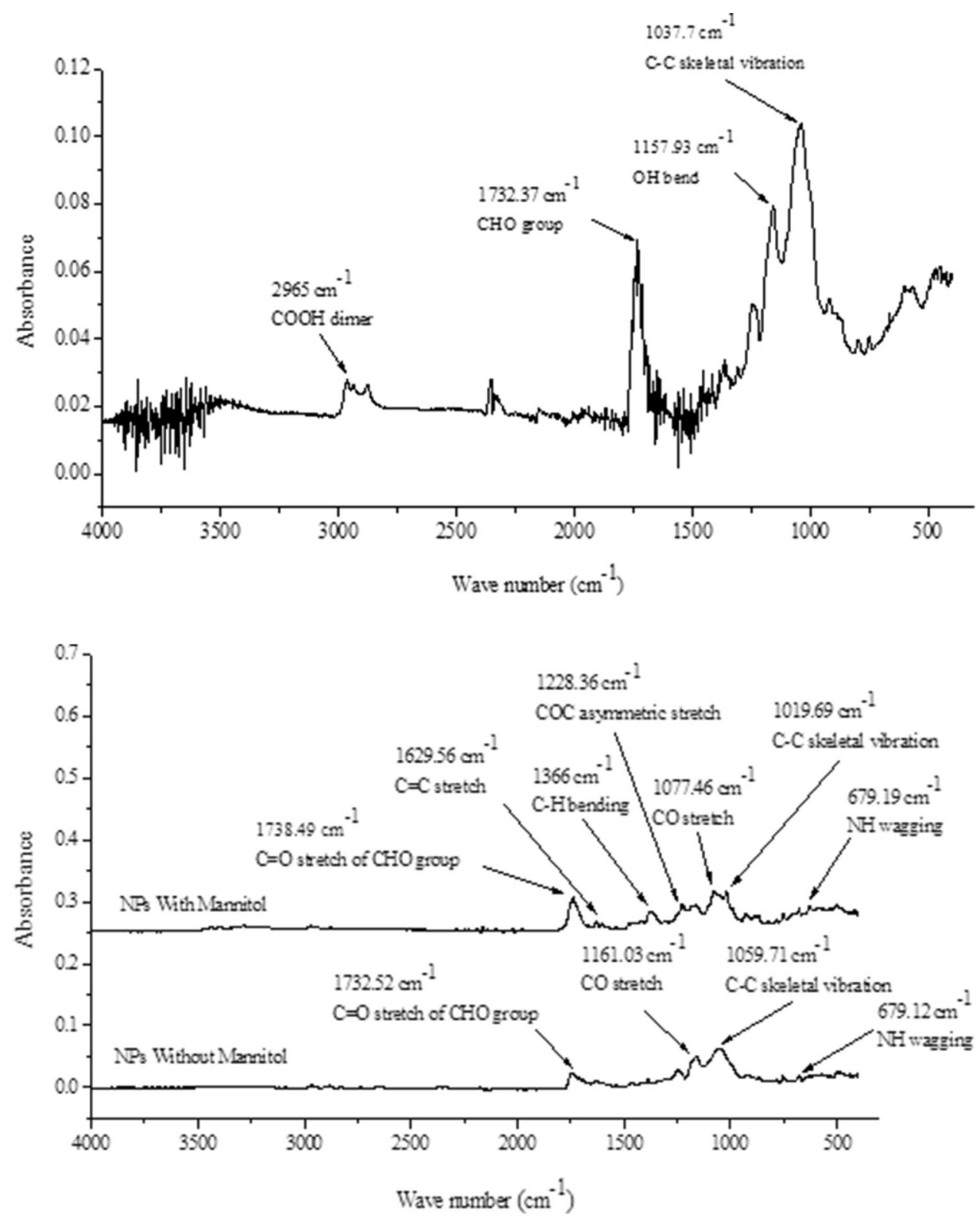

masked. That may have been the reason for no peaks in the above region. From the FT-IR analysis of pure drug, polymer and polymer drug nanoparticles, spectra confirmed the incorporation of both $\mathrm{CMCAB}$ and $\mathrm{ACV}$ in nanoparticles.

Differential scanning calorimetry study

DSC studies were carried out on ACV-CMCAB nanoparticles along with pure $\mathrm{ACV}$ and $\mathrm{CMCAB}$ to assess their phase transition behavior. In the case of pure $\mathrm{ACV}$ which is crystalline in nature, no changes in heat flow were observed up to temperature of approximately $75{ }^{\circ} \mathrm{C}$. After this, heat flow started to increase at a slow rate in the sample with temperature and reached a maximum of nearly $22 \mathrm{~mW}$ at $109.56{ }^{\circ} \mathrm{C}$ which corresponds to its glass transition temperature $\left(T_{\mathrm{g}}\right)$. After this, no much difference is observed in enthalpy of the sample and was constant in the temperature range of $125-250{ }^{\circ} \mathrm{C}$. As the temperature increased further, endothermic heat flow suddenly increased to a value of nearly $42 \mathrm{~mW}$ and a sharp peak was observed at temperature of $258.89^{\circ} \mathrm{C}$. That corresponds to the melting temperature $\left(T_{\mathrm{m}}\right)$, and this clearly showed the phase transition behavior of pure $\mathrm{ACV}$ from solid to liquid state. DSC thermogram of ACV is shown in Fig. 10.

For CMCAB, which is an amorphous polymer, endothermic heat flow started to vary from initial 
Fig. 10 DSC thermogram of as-received $\mathrm{ACV}$

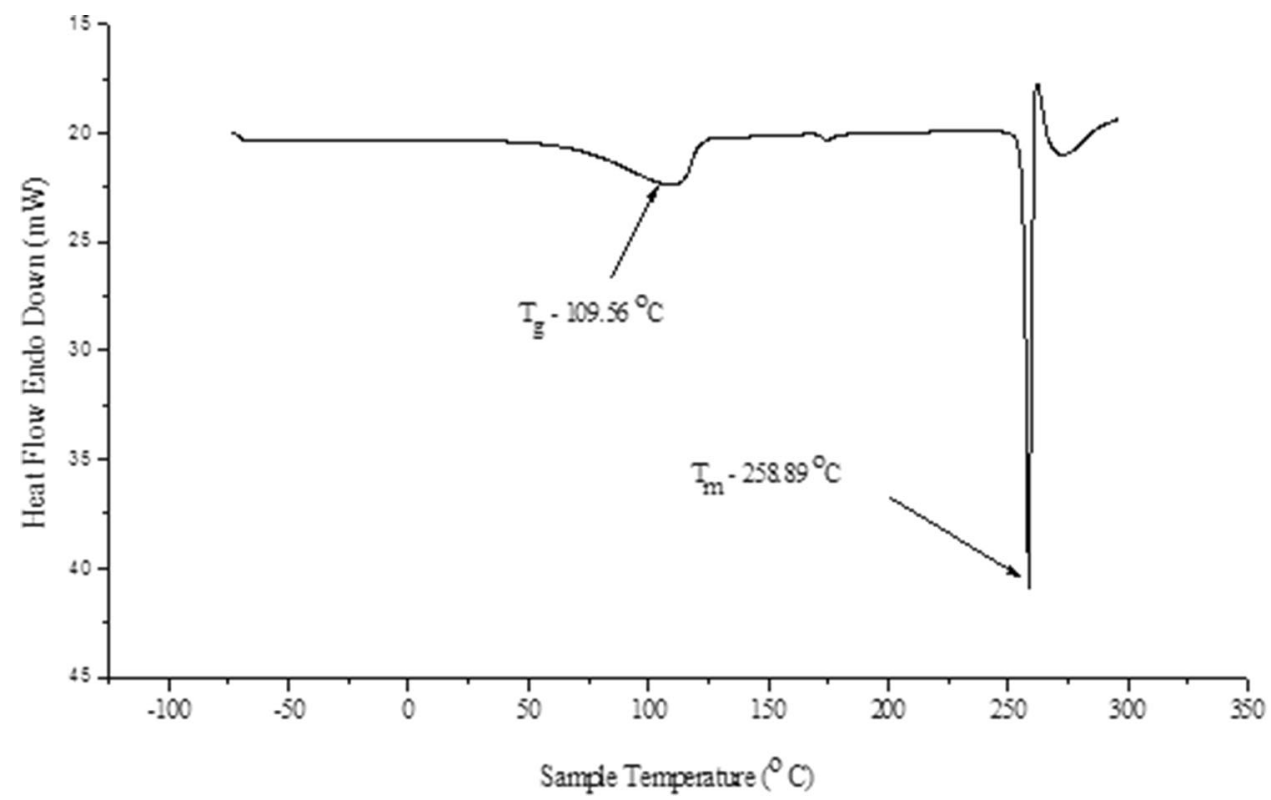

temperature of $-70^{\circ} \mathrm{C}$. Not alike in the case of $\mathrm{ACV}$, heat flow was not constant even in small temperature ranges and varied with temperature throughout the analysis. Though changes in endothermic enthalpy were observed in the temperature ranges of 0-50, 125-150 and 225-275 ${ }^{\circ} \mathrm{C}$, they were not that large, suggesting the poor mobility of polymer chains. Regarding the polymer powder, the broad first heat endotherm $\left(50-100^{\circ} \mathrm{C}\right)$ could be related to moisture loss. The polymer powder first-heat shows a step change near $150{ }^{\circ} \mathrm{C}$, as in a glass transition; but this is complicated by a minor, overlapping exotherms ( $\sim 160$ and $\left.180{ }^{\circ} \mathrm{C}\right)$.

The complicating exotherms may be because of an interchain esterification (crosslinking), or it could be related to crystallization - the true origin of this peak is unknown. Of the mentioned three temperature ranges, slope of curve is larger in the case of $125-150{ }^{\circ} \mathrm{C}$ temperature range and this clearly indicates the second-order glass transition temperature $\left(T_{\mathrm{g}}\right)$, which was noticed at $140.6{ }^{\circ} \mathrm{C}$. No sudden peaks were observed as in the case of ACV. DSC thermogram of CMCAB is shown in Fig. 11. In the case of ACV-CMCAB nanoparticles, the study confirmed that polymer drug nanoparticles were stable up to temperature range of $150-160{ }^{\circ} \mathrm{C}$. No sharp peaks or large changes were observed in terms of heat endotherms up to this temperature.

The initial exotherms as in the case of pure $\mathrm{CMCAB}$ powder in the temperature range of $50-100{ }^{\circ} \mathrm{C}$ were because of moisture loss. For nanoparticles with mannitol, a sharp peak has been observed at temperature $162.9{ }^{\circ} \mathrm{C}$ and the same is not present in the nanoparticles without mannitol. This peak according to the literature (Barbara 2004) is the melting temperature of pure mannitol and suggests its crystalline behavior. The curve is smooth in the case of nanoparticles without mannitol suggesting amorphous behavior and the glass transition temperature is approximately $166.2^{\circ} \mathrm{C}$. This might be due to inhibition of crystallization of $\mathrm{ACV}$ during nanoparticle formation by the polymer. So, it can be concluded that ACV in the nanoparticles was in an amorphous or a solid solution state in the polymer matrix after the fabrication. DSC thermograms of ACVCMCAB nanoparticles with and without mannitol addition are shown in Fig. 12.

\section{Drug loading}

In order to obtain the actual amount of drug present in polymer drug nanoparticles, drug loading percentage was determined by spectrophotometric method for nanoparticles. Absorbance values of previously prepared nanoparticle solution were determined at fixed wavelength of $253 \mathrm{~nm}$. This value is substituted in the standard curve of ACV to get corresponding experimental concentration. Ratio of experimental and actual concentrations gives the percentage of drug present in nanoparticles. Absorbance and percentage drug loaded for both nanoparticle batches are tabulated in Table 3.

Drug loading in the both the cases is comparable and slightly higher in case of flash nanoprecipitation. It is to be noted that mass concentration of drug in water was kept constant as $0.3703 \mathrm{mg} / \mathrm{ml}$ in both cases. However, there was higher entrapment of the drug in the polymer during rapid mixing in the MIVM.

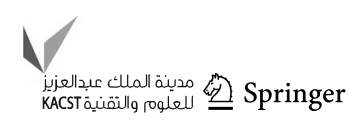


Fig. 11 DSC thermogram of pure $\mathrm{CMCAB}$

Fig. 12 DSC plot of ACVCMCAB nanoparticles with and without mannitol
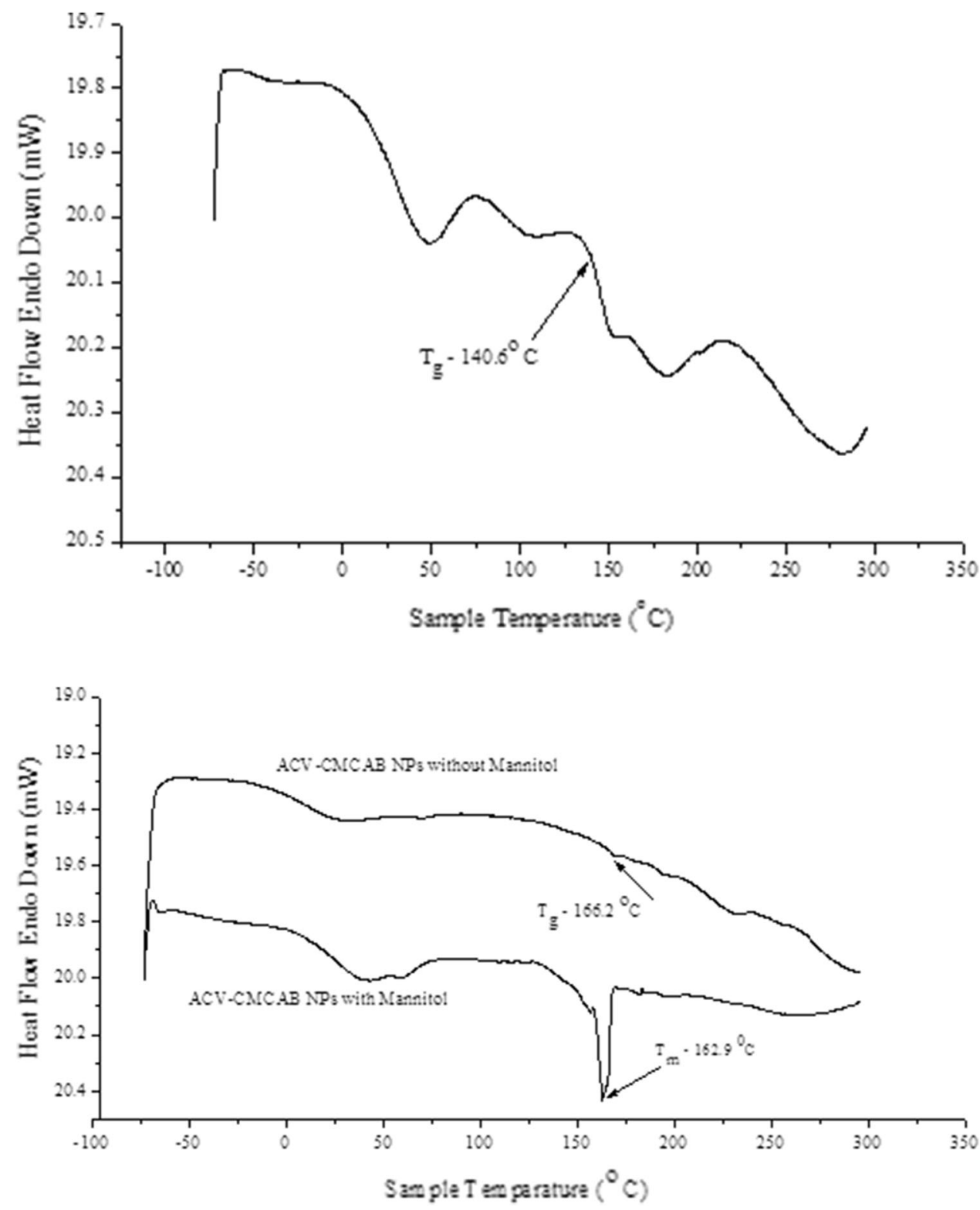

In vitro studies

Drug release profiles from in vitro studies at $\mathrm{pH} 6.8$ for nanoparticles and pure drug with absorbance values are shown in Fig. 13. From the profiles, it is clearly observed that release percentage of ACV from nanoparticles (nearly $55 \%$ ) was more than pure drug (nearly $5 \%$ ) over a period of time by tenfolds. The increase in the solution concentration of the drug was observed for nanoparticles compared to the as-received crystalline drugs. Reducing the particle size of the ACV from that of the as-received powder greatly increases the specific surface area, which provides an additional method to enhance the dissolution of ACV. Also, CMCAB was effective in entrapping the drug in amorphous form to enhance drug release.
The residence time of particles in small intestine is approximately 3-4 h. The aim is to release most of the drug in the small intestine. Higher concentration of drug would lead to higher absorption. Rapid release of ACV in small intestine could lead to reduced dose and high bioavailability. The dissolution rate could be increased by increasing the surface area which could be increased by decreasing the size. As shown, the nanoparticles have shown higher release and solution concentration of drug in the intestinal fluid. As the residence time is only 3-4 h, a longer study is not desirable. However, the experiment is redone for few more hours to investigate any change in the drug release from nanoparticles. The results clearly showed that there is no significant release after the fifth hour. 
Table 3 Drug loading (wt $\%$ ) of ACV-CMCAB nanoparticles prepared by simple precipitation and flash nanoprecipitation

\begin{tabular}{llc}
\hline S. no & Particle preparation method & $\begin{array}{l}\text { wt\% drug loaded } \pm \text { standard } \\
\text { deviation from three batches }\end{array}$ \\
\hline 1 & Simple precipitation & $8.5 \pm 2$ \\
2 & Flash precipitation & $9.24 \pm 0.5$ \\
\hline
\end{tabular}

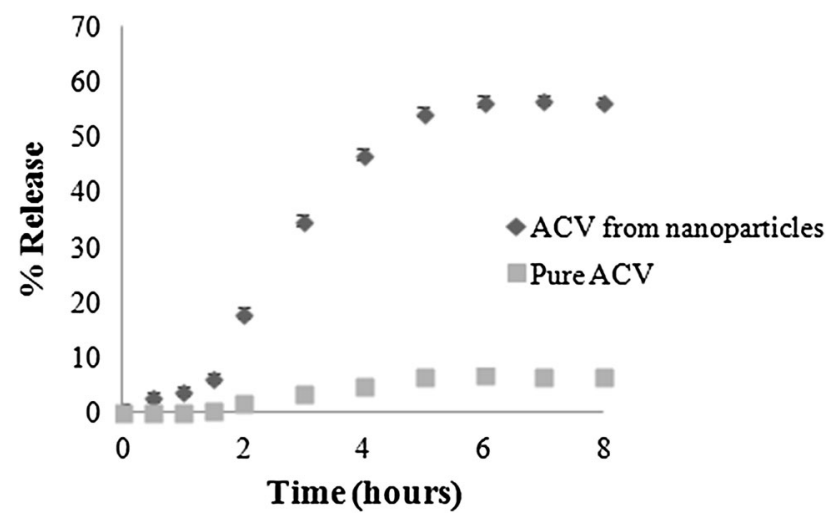

Fig. 13 Dissolution profiles of pure ACV and ACV-CMCAB nanoparticles in phosphate buffer of $\mathrm{pH} 6.8$ (Standard Deviation error of 1)

\section{Conclusion}

This study demonstrates a method for producing CMCABACV nanoparticles. Two methods were used to prepare the nanoparticles: first, by simple precipitation method and second, by flash nanoprecipitation method using a multiinlet vortex mixer. Well-defined particle sizes were obtained with MIVM between 100 and $500 \mathrm{~nm}$ and drug loading of $9.2 \mathrm{wt} \%$. The drug loading efficiencies in the final particles were approximately $40 \%$. CMCAB polymer was effective in entrapping the drug in its amorphous state in the particle and this increased the solubility of the drugs in buffer solution. The nanometer size greatly increased specific surface area which greatly enhanced dissolution of $\mathrm{ACV}$ and higher release (tenfolds compared to pure ACV).

However, these are the preliminary experiments on the effectiveness of the $\mathrm{CMCAB}-\mathrm{ACV}$ nanoparticles and more complete and statistically valid tests need to be performed. More investigation is needed to characterize the very long storage stability of these particles. The enhancement of release of drugs from polymer nanoparticles for effective drug delivery of low solubility antiviral drugs is an attractive area for additional research.

Acknowledgments The authors gratefully acknowledge funding and support from the Research Initiation Grant, Birla Institute of Technology and Science, Pilani. The authors also thank Prof. R. N. Saha, Director, BITS Pilani, Dubai, for providing the drug ACV and valuable inputs to conduct the experiments. The authors also thank
Mr. Subodh Azad, Department of Chemical Engineering, for helping with FTIR and DSC experiments.

Open Access This article is distributed under the terms of the Creative Commons Attribution License which permits any use, distribution, and reproduction in any medium, provided the original author(s) and the source are credited.

\section{References}

Ahmed HE, Amany OK, Gehanne ASA (2010) Ammonium methacrylate units polymer content and their effect on acyclovir colloidal nanoparticles properties and bioavailability in human volunteers. Colloid Surf B 75(2):398-404

Amany OK, Gehanne ASA, Ahmed SG, Nahed DM (2009) Preparation of intravenous stealthy acyclovir nanoparticles with increased mean residence time. AAPS PharmSciTech 10(4):1427-1436

Ángel G, Juan JF, Ferran B, Rosa C, Ángel T, Miguel Q (1999) Synthesis and structural characteristics of metal-acyclovir (ACV) complexes: $\quad\left[\mathrm{Ni}(\right.$ or $\left.\mathrm{Co})(\mathrm{ACV})_{2}(\mathrm{H} 2 \mathrm{O})_{4}\right] \mathrm{Cl}_{2} .2 \mathrm{ACV}$, $\left[\mathrm{Zn}(\mathrm{ACV})-\mathrm{Cl}_{2}\left(\mathrm{H}_{2} \mathrm{O}\right)\right], \quad\left[\mathrm{Cd}(\mathrm{ACV}) \mathrm{Cl}_{2}\right] \cdot \mathrm{H}_{2} \mathrm{O}$ and $[\{\mathrm{Hg}(\mathrm{ACV})$ $\left.\mathrm{Cl}_{2}\right\} x$ ]. Recognition of acyclovir by $\mathrm{Ni}-\mathrm{ACV}$. J Chem Soc Dalton Trans 2:167-173

Antona JW, Diana F, Karen LG (1994) Acyclovir. Drugs 47(1):153-205

Barbara HS (2004) Organic molecules, Chapter 4 In: Infrared spectroscopy-fundamentals and applications. Wiley, Sussex, pp. 71-93

Barbara S, Silvia A, Flavio R, Susi B, Nadia N, Silvia T, Patrizia C, Luigi C (2012) Nonpolymeric nanoassemblies for ocular administration of acyclovir: pharmacokinetic evaluation in rabbits. Eur J Pharm Biopharm 80:39-45

Ben F (1997) Infrared spectroscopy, chapter 3. In: Modern chemical techniques. The Royal Society of Chemistry, London, UK, pp 62-92

Bernard BG, Florent P, Yves C, Helene P (2009) Novel nanoparticles made from amphiphilic perfluoroalkyl-cyclodextrin derivatives: preparation, characterization and application to the transport of acyclovir. Int J Pharm 375(1-2):155-162

Bin L, Stephanie K, Kim H, Lindsay W, Lynne ST, Kevin JE (2013) Solid dispersion of quercetin in cellulose derivative matrices influences both solubility and stability. Carbohydr Polym 92(2):2033-2040

Biswajit M, Balaram P, Buddhadev L, Arup M (2007) Sustained release of acyclovir from nano-liposomes and nano-niosomes: an in vitro study. Int J Nanomedicine 2(2):213-225

Boris R, Ying L, Robert KP (2010) Optimized descriptive model for micromixing in a vortex mixer. Chem Eng Commun 197(8): $1068-1075$

Claudia G, Claudio B, Adriana M, Donatella P, Maria AV, Giovanni P, Vincent HLL, Massimo F (2003) Influence of preparation conditions on acyclovir-loaded poly- $d, l$-lactic acid nanospheres and effect of PEG coating on ocular drug bioavailability. Pharm Res 20(4):584-590

David L, Shankar S, Manuela D, Andrea C, Linda P, Dino A, Pradeep V, Francesco T, Roberta C (2013) Encapsulation of acyclovir in new carboxylated cyclodextrin-based nanosponges improves the agent's antiviral efficacy. Int J Pharm 443(1-2):262-272

Dembri A, Montisci M-J, Gantier J, Chacun H, Ponchel G (2001) Targeting of $3^{\prime}$-Azido $3^{\prime}$-deoxythymidine (azt)-loaded poly(isohexylcyanoacrylate) nanospheres to the gastrointestinal mucosa and associated lymphoid tissues. Pharm Res 18(4):467-473 
Deng-Guang Y, Li-Min Z, Christopher J, Branford W, Jun-He Y, Xia W, Ying L, Wei Q (2011) Solid dispersions in the form of electrospun core-sheath nanofibers. Int $\mathrm{J}$ Nanomedicine 6:3271-3280

Destache C, Belgum T, Christensen K, Shibata A, Sharma A, Dash A (2009) Combination antiretroviral drugs in PLGA nanoparticle for HIV-1. BMC Infect Dis 9(1):198

Duan J, Zhang Y, Han S, Chen Y, Li B, Liao M, Chen W, Deng X, Zhao J, Huang B (2010) Synthesis and in vitro/in vivo anticancer evaluation of curcumin-loaded chitosan/poly(butyl cyanoacrylate) nanoparticles. Int J Pharm 400(1-2):211-220

Elaine MM, Gary GL, Eugene RC (2003) Nanosizing: a formulation approach for poorly-water-soluble compounds. Eur J Pharm Sci 18(2):113-120

Esther EF, Helen AW, Judith RG, Pamela LC, James AW, Richard JH (2006) Herpes simplex virus 2 infection increases HIV acquisition in men and women: systematic review and meta-analysis of longitudinal studies. AIDS 20(1):73-83

Florent P, Marine D, Yves C, Helene P (2013) Design, synthesis, and in vitro evaluation of new amphiphilic cyclodextrin-based nanoparticles for the incorporation and controlled release of acyclovir. Eur J Pharm Biopharm 83(1):25-32

Ga de Jalona E, Blanco-Prietoa MJ, Ygartuaa P, Santoyob S (2003) Increased efficacy of acyclovir-loaded microparticles against herpes simplex virus type 1 in cell culture. Eur $\mathrm{J}$ Pharm Biopharm 56(2):183-187

Hak-Kim C, Philip CLK (2011) Production methods for nanodrug particles using the bottom-up approach. Adv Drug Deliv Rev 63(6):406-416

Hariharan S, Bala I, Kumar MNVR (2004) PLGA nanoparticles in drug delivery: the state of the art. Crit Rev Drug Carr Syst 21(5):387-422

Ismail AA, Sanaa AE, Medhat AF, Ahmed MD (2007) Influence of a niosomal formulation on the oral bioavailability of acyclovir in rabbits. AAPS PharmSciTech 8(4), Article 106

Janine CC, Michael GO, Rodney OF (2009) A microscale multi-inlet vortex nanoprecipitation reactor: turbulence measurement and simulation. Appl Phys Lett 94:204104

Jessica DP, Thelma LW, Wilson AK, Kevin JE, Michael CS, Larry RL Jr (2007) Zero-order release formulations using a novel cellulose ester. Cellulose 14:73-83

Junia MP, Raquel M, Grace AI, Heather EM, Sriranganathan N, Lynne ST, Richey MD, Kevin JE (2013) Interplay of degradation, dissolution and stabilization of clarithromycin and its amorphous solid dispersions. Mol Pharm 10(12):4640-4653

Katharine JL, Geoffrey PG, George PS (2008) An estimate of the global prevalence and incidence of herpes simplex virus type 2 infection'. Bull World Health Organ 86(10):805-812

Lei Q, Ziqiang S, Mingshan Y, Wenjun W, Feijun W, Long X, Shaoyi L, Yunhua Z (2013) Electrospun carboxymethyl cellulose acetate butyrate (CMCAB) nanofiber for high rate lithium-ion battery. Carbohydr Polym 96(1):240-245

Li B, Kim H, Lindsay W, Lynne ST, Kevin JE (2013) Stability and solubility enhancement of ellagic acid in cellulose ester solid dispersions. Carbohydr Polym 92(2):1443-1450
Löbenberg R, Maas J, Kreuter J (1998) Improved body distribution of 14C-labelled AZT bound to nanoparticles in rats determined by radioluminography. J Drug Target 5(3):171-179

Mainardes RM, Evangelista RC (2005) PLGA nanoparticles containing praziquantel: effect of formulation variables on size distribution. Int J Pharm 290(1-2):137-144

Mainardes RM, Gremião MPD, Brunetti IL, Da Fonseca LM, Khalil NM (2009) Zidovudine-loaded PLA and PLA-PEG blend nanoparticles: influence of polymer type on phagocytic uptake by polymorphonuclear cells. J Pharm Sci 98(1):257-267

Michael CS, Jessica DP, Larry L, Shane KK, Sandra K, Kevin JE (2009) Enhanced dissolution of poorly soluble drugs from solid dispersions in carboxymethyl cellulose acetate butyrate matrices. In: Edgar K, et al. (eds) Polysaccharide materials: performance by design, ACS symposium series. American Chemical Society, Washington

Peng Y, Thomas B (2008) Characterization of D-mannitol by thermal analysis, FTIR, and raman spectroscopy. Am Lab 40(14):24-27

Raval JP, Naik DR, Amin KA, Patel PS (2011) Controlled-release and antibacterial studies of doxycycline-loaded poly( $\varepsilon$-caprolactone) microspheres. J Saudi Chem Soc (in press)

Ravi ST, Swapan KS, Ripal G, Ashim KM (2008) Synthesis, metabolism and cellular permeability of enzymatically stable dipeptide prodrugs of acyclovir. Int J Pharm 361(1-2):118-124

Sandra K (2009) Polysaccharides in oral drug delivery? Recent applications and future perspectives. In: Polysaccharide materials: performance by design. J Am Chem Soc 1017:13-30

Sharma P, Garg S (2010) Pure drug and polymer based nanotechnologies for the improved solubility, stability, bioavailability and targeting of anti-HIV drugs. Adv Drug Deliv Rev 62(4-5): 491-502

Suwussa B, Zilong Z, Tao C, Lin W, Chunmei L, Ting F, Weihong T (2012) Nanotechnology in therapeutics. Nanomedicine 7(8):1253-1271

Vivek K, Meenakshi B, Harish D, Deepak K (2014) Nanoparticle technology for the delivery of poorly water-soluble drugs. Pharm Technol, pp 1-11

Wang BH, Yu CY, Wang XZh (2002) Supercritical drying of nanometer porous materials. In: Proceedings of the 8th national drying symposium, January 8-10, pp 22-31, Harbin, China

Yanxiang S, Janine CC, Rodney OF, Michael GO (2013) Measurements of turbulence in a microscale multi-inlet vortex nanoprecipitation reactor. J Micromech Microeng 23(7):075005

Ying L, Chungyin C, Ying L, Robert KP, Rodney OF (2008) Mixing in a multi-inlet vortex mixer (MIVM) for flash nano-precipitation. Chem Eng Sci 63(11):2829-2842

Zane AB, Stacy S, Judy Z, Jerome K, Arthur M, Rhoda LA, Heather WD, Sylvia B, Millie H, Lawrence C (1997) The acquisition of herpes simplex virus during pregnancy. $\mathrm{N}$ Engl $\mathrm{J}$ Med 337(8):509-515 